\title{
Urban Gardening in the Crisis Conjuncture
}

\section{Christopher Maughan*}

Institute of Advanced Study, University of Warwick

*Correspondence: chris.maughan@coventry.ac.uk

\begin{abstract}
Urban gardening finds itself at a juncture - not only are crises caused and exacerbated by the industrial food system urgently demonstrating the need for more localised, sustainable, and democratically-determined food systems, but alternative food movements are increasingly negotiating crises of their own. Critical Foodscapes was a one-day conference part-funded by Warwick's Institute of Advanced Study (IAS) and the Food GRP. The conference was put together with the intention of bringing a 'critical studies' approach to the emerging research area of urban community food growing; namely, to put critical - but constructive - pressure on some of the assumptions which underlie current theory and practice of the various forms of urban food growing. This article offers some reflections on the conference itself as well as on the prospects for urban gardening more generally.
\end{abstract}

Keywords: urban gardening; urban agriculture; agroecology; food systems; food sovereignty; sustainability

Funding: See page 171

Peer review: This article has been subject to a double blind peer review process

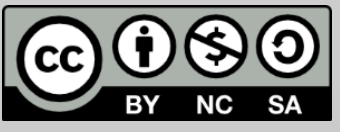

C Copyright: The Authors. This article is issued under the terms of the Creative Commons Attribution NonCommercial Share Alike License, which permits use and redistribution of the work provided that the original author and source are credited, the work is not used for commercial purposes and that any derivative works are made available under the same license terms.
Urban gardening has long promised fairer and more sustainable alternatives to industrialised food production and the organisation of modern urban spaces. However, in the Global North, despite recent increases in popularity and a conspicuous proliferation of its forms, urban gardening appears to have had minimal material or cultural influence on how we eat or how we live. As Holt-Gimenez (2016) puts it, 'if these innovations are so good why isn't everyone doing them?' While the reasons for urban gardening's shortcomings are certainly numerous, for Holt-Gimenez, a key factor is the failure of the alternative food movement to adequately address issues of social justice. He is not alone; indeed, increasing emphasis is now being placed on the 'social exclusionary and class-based nature of alternative food systems', and that the fact that, for example, the 'immiserating and poor health of farm workers is a major feature not only of cheap industrial foods, but of higher value niches' (Dixon 2016: 1).

Urban gardening thus finds itself at a juncture - not only are crises caused and exacerbated by the industrial food system urgently 
demonstrating the need for more localised, sustainable, and democratically-determined food systems, but alternative food movements are increasingly negotiating crises of their own. As Michel Pimbert (2006: 34) points out, 'citizen spaces' - even the ones which have been conceived explicitly to address issues of social and environmental justice - 'are infused with power relations, affecting who enters them, who speaks with what knowledge and voice, and who benefits'. It remains to be seen if urban gardening can - on the one hand - form part of a viable alternative to the industrial food system and - on the other - develop the critical insight to ensure it can identify and excise the unjust operation of power in its own governance.

Critical Foodscapes was a one-day conference part-funded by Warwick's Institute of Advanced Study (IAS) and the Food GRP. The conference was put together by Dr Chris Maughan (IAS Early Career Fellow) with the intention of bringing a 'critical studies' approach to the emerging research area of urban community food growing; namely, to put critical but constructive - pressure on some of the assumptions which underlie current theory and practice of the various forms of urban food growing.

Conference papers addressed such issues as the emerging (and as yet incomplete) evidence base for urban gardening's social and environmental outputs; the extent to which forms of urban gardening can exacerbate processes of 'gentrification'; the effectiveness of 'urban food strategies' and policies for extending the reach and impact of community food growing; and how to better organise community growing initiatives at the grassroots level.

A sense of the sheer complexity of this task was given by the conference keynote, Chiara Tornaghi (Centre for Agroecology Water and Resilience) who described the obstacles common in, what she called, 'the food disabling city'. According to Tornaghi, 'urban agroecology' is structurally discouraged and prevented in today's developed cities: obstacles to an urban food grower might include absurd aesthetic standards (like mandatory lawn fronts on suburban properties), inappropriate regulations (e.g. restrictions pertaining to water access and composting, or prohibitively high rents), and even tall buildings that can fatally restrict a garden's access to sunlight. Our response as activists and scholars, Tornaghi suggests, should be to stop considering such a condition as 'normal', and instead to claim one's 'right to the city' and to an expansive form of 'urban metabolism'.

Tornaghi's recommendations were very much geared towards effecting a cultural shift in how we approach urban food growing and cities in general, rather than making specific recommendations. While generalisms are of course a common (not to mention very useful) feature 
of keynote addresses, Tornaghi's approach reflects a deeper point about the place-specific nature of urban gardens; indeed, as Tornaghi reminded us, 'there is no recipe' for effective urban agroecology, this can only really be worked out in situ.

Tornaghi's conceptual overview was complemented, in fact, by the specificity of other talks; we heard, for example, from grassroots organisers and participants such as Pam Warhurst (Incredible Edible); Jayne Bradley (Edible Eastside), and Chesta Tiwari (Good Food Oxford) who spoke about the challenges they have faced in developing their projects. Warhurst offered a characteristically optimistic account of the Incredible Edible project, citing the simple power of 'conversations' in fostering changes in participant horizons of possibility, not only in terms of food growing, but also their personal, civic, and collective capacities. While just as enthusiastic, Tiwari's talk focused on the organisational problems common to community gardens. According to Tiwari, more often than not community gardens rely on unsustainable forms of governance (i.e. one or two people 'doing all the work'). Tiwari's talk offered yet another reminder that while urban gardens can make positive changes in nutritional and environmental terms, they are primarily 'all about people'; if they don't work on a social level, they won't work on any level (at least not for very long).

The role of policy in urban gardening was discussed by a number of speakers, among them Rebecca Marshall (ex-Community Land Advisory Service), Lorenza Sganzetta (Milan) and Hannah Pitt (Cardiff University), all of whom remained wary of the capacity of policy on its own to effect the desired changes in contemporary food systems. The final keynote by Jeremy lles (ex-CEO FCFCG), echoed a similar sense (apparent, in fact, throughout the conference) of the sheer scale of the challenges faced by proponents of urban gardening, whether in terms of health (the UK now boasts a $60 \%$ adult obesity rate), inadequate infrastructure, an unfavourable policy environment, or the increasing volatility of the climate itself.

During the final panel session of the day, Anton Rosenfeld (Garden Organic), two representatives from 'Growhampton', and Susan Haedike (Warwick) brought a compellingly human touch to proceedings. Rosenfeld in particular offered valuable insight into the rich history of urban gardening on the part of immigrant growers, itemising the scores of unusual or 'exotic' edible plants he had found growing on allotment sites across the UK, as well as the tragic fragility of such practices. Many techniques and plant varieties are very close to disappearing entirely, either as a result of knowledge not being 'passed on' or the dominance and homogenising effects of mainstream horticultural suppliers. 
Though in general Rosenfeld's talk was positively received, his use of the word 'exotic' to describe the plant varieties grown by immigrants did not go unchallenged. While Rosenfeld acknowledged the problems with the term, he cited the difficulty of finding an alternative term that was also recognised by the individuals with whom he is working. The issue clearly warrants further attention, though also provided yet further evidence of the profoundly complex cultural contexts that must be navigated by urban food researchers and activists, and that critical scrutiny must be directed inwards as much as outwards.

Perhaps the most resolutely 'critical' paper of the conference came from Brian Elliott (Portland State) who discussed the effects of urban agriculture in Portland, Oregon - described by him as 'the most gentrified city in the US'. Portland emblematises both the rapid expansion enjoyed by urban agriculture in recent times - 'urban agriculture', Elliott told us, 'suffuses the city' with over 100 community gardens in operation - but also its often unwitting participation in the city's 'uneven development'. The alternative food movement has become increasingly associated with the processes of 'green gentrification'; that is, where environmentally prestigious developments (like community gardens, but also organic food retailers, parks, and other 'eco-friendly' infrastructure) are used as pretexts to increase rents, thereby displacing lower-income residents. Added to this, Elliott suggested, urban gardeners can often be oblivious to the exclusionary mechanisms their practice encodes, asking 'for whom is agriculture a celebratory activity, and for whom does it conjure a traumatic memory?' Those with an ancestral history of plantation-based slavery or of territorial dispossession must be considered if urban gardens ever expect to achieve the multi-cultural participation we have so long desired.

'Green gentrification' remains a huge problem for urban agroecology; I wish to suggest, however, that along with other tensions, problems, and challenges faced by urban food growers, the issue of gentrification offers a powerful reminder of the value of a robust critical introspection. Gentrification can seem like an ontological feature of urban development - i.e. if you do something good the parasitic forces of capitalism will always exploit that achievement for profit; while this is undoubtedly a troubling feature of the capitalist political economy, we must remember that the strength of urban gardening is its ability to refocus attention on people, as well as their relationships with the land and each other.

Projects that remain attentive to the social, cultural, environmental, and historical contexts in which they are situated - in short, the lived experience of people that make up that community and not some abstract notion of environmental prestige - will be the most enduring 
and impactful projects, as well as the least susceptible to unwitting participation in processes of gentrification. In practice this means prioritising good planning and organisation, a culture of self-appraisal and critique, and the aspiration at least to develop a culture of politicised conversation about the meaning and implications of what we do as urban gardeners. This conference provided ample evidence - to me at least that this is the direction we are travelling in.

For a full list of talks please see the conference programme. Most talks were recorded and are available in the links below, as are some of the conference presentations. Furthermore, conference proceedings are being developed into a special issue of Renewable Agriculture and Food Systems, due to be published in the spring of 2017.

\section{Acknowledgements}

Thanks go to the Institute of Advanced Study, Warwick and the Warwick Food GRP for joint funding this conference.

\section{Critical Foodscape Conference Recordings}

Conference page (Overview)

\begin{tabular}{l|l|l} 
Intro/Keynote 1 - Dr Chiara Tornaghi & Panel 1 - Pam Warhurst & Rebecca \\
\hline
\end{tabular} Marshall 1 Dr Brian Elliott

\begin{tabular}{l|l|l|l} 
Panel 2a 'Sustainability' & Dr George Martin & Jayne Bradley & Dr Marion
\end{tabular} Bonow

Panel 3a 'Organisation' / Keynote 2 - Chesta Tiwari 1 Dr Ute Kelly | Ian Humphreys | Jeremy lles 


\section{References}

Dixon, J. (2016), 'Critical nutrition studies within critical agrarian studies: a review and analysis', The Journal of Peasant Studies, $0(0), 1-9$, https://doi.org/10.1080/03066150.2016.1198513.

Holt-Giménez, E. (2016), 'Food movements, climate resilience, social change', TEDxBerkeley Talks, https://www.youtube.com/watch?v=fX9dV9zCN-A.

Pimbert, M. P. (2006), Transforming knowledge and ways of knowing for food sovereignty, London: IIED.

To cite this article:

Maughan, C. (2016). Urban Gardening in the Crisis Conjuncture. Exchanges:

The Warwick Research Journal, 4(1), 167-172. Retrieved from:

http://exchanges.warwick.ac.uk/index.php/exchanges/article/view/131 